\title{
FACTORIZATION INDICES FOR MATRIX POLYNOMIALS
}

\author{
BY I. GOHBERG ${ }^{1}$, L. LERER AND L. RODMAN \\ Communicated by R. G. Douglas, August 8, 1977
}

Let $\Gamma$ be a rectifiable simple closed contour in the complex plane $\mathbf{C}$ bounding the domain $F^{+}$. The notation $F^{-}$will be used for the complement to $F^{+}$ $\cup \Gamma$ in $C \cup \infty$. It will always be assumed that $\lambda=\infty \in F^{-}$.

A polynomial $L(\lambda)=A_{0}+\lambda A_{1}+\cdots+\lambda^{m} A_{m}$ with $n \times n$ matrix coefficients $A_{j}$ and with det $L(\lambda) \neq 0$ for $\lambda \in \Gamma$ is said to admit a (right standard) factorization relative to the contour $\Gamma$ in case

$$
L(\lambda)=L_{+}(\lambda) D(\lambda) L_{-}(\lambda),
$$

where $L_{+}(\lambda)$ is a matrix polynomial with det $L_{+}(\lambda) \neq 0$ for $\lambda \in F^{+} \cup \Gamma, L_{-}(\lambda)$ is a matrix polynomial in the variable $(\lambda-a)^{-1}$ for some $a \in F^{+}$and $\operatorname{det} L_{-}(\lambda)$ $\neq 0$ for $\lambda \in F^{-}$, and

$$
D(\lambda)=\operatorname{diag}\left((\lambda-a)^{\kappa_{1}},(\lambda-a)^{\kappa_{2}}, \ldots,(\lambda-a)^{\kappa} n\right)
$$

with some nonnegative integers $\kappa_{1} \leqslant \kappa_{2} \leqslant \cdots \leqslant \kappa_{n}$. Such a factorization of $L(\lambda)$ is not unique, but the numbers $\kappa_{1} \leqslant \kappa_{2} \leqslant \cdots \leqslant \kappa_{n}$ which are called the (right) partial indices are uniquely determined by $L(\lambda)$ (see [1], [5]). An analogous definition of left standard factorization is possible. Here we deal only with right factorization.

The factorization indices play an important role in the theory of systems of singular integral equations (see [1], [5]), Wiener-Hopf equations, partial differential equations, and the classification of holomorphic vector bundles on the Riemann sphere. There exists an algorithm for computing the indices which is described in [1].

In this paper we obtain some explicit formulas for the partial indices. As a main tool, we use the ideas of spectral analysis of matrix polynomials developed in [2], [3], [4].

To begin with, consider the linear case $L(\lambda)=A-\lambda$. Let $A=$ $\operatorname{diag}\left(A_{1}, A_{2}\right)$ be a block representation where the eigenvalues of $A_{1}$ are inside $F^{+}$ and the eigenvalues of $A_{2}$ are inside $F^{-}$. Then (assuming that $\lambda=0$ is inside $F^{+}$)

AMS (MOS) subject classifications (1970). Primary 30A88, 45E05, 45E10; Secondary $15 \mathrm{~A} 18$.

Key words and phrases. Factorization, partial indices, boundary value problem for analytic function, matrix valued polynomials.

1 Partial supported by NSF grant at State University of New York at Stony Brook. Copyright O 1978, American Mathematical Society 
the equality

$$
A-\lambda I=\operatorname{diag}\left(I, A_{2}-\lambda I\right) \operatorname{diag}(\lambda I, I) \operatorname{diag}\left(\lambda^{-1} A_{1}-I, I\right)
$$

is a factorization of $A-\lambda I$ and the indices are $(0,0, \ldots, 0,1,1, \ldots, 1)$ where the number of ones is exactly equal to $\operatorname{rank} A_{1}\left(=\operatorname{rank} \operatorname{Im} \int_{\Gamma}(A-\lambda)^{-1} d \lambda\right)$. For a matrix polynomial, this result can be generalized as follows.

Theorem 1. Let $L(\lambda)=A_{0}+\lambda A_{1}+\cdots+\lambda^{m} A_{m}$ be a matrix polynomial with det $L(\lambda) \neq 0$ for $\lambda \in \Gamma$. Then for the factorization indices $\kappa_{1} \leqslant \kappa_{2}$ $\leqslant \cdots \leqslant \kappa_{n}$ of $L(\lambda)$ the following equalities hold

$$
\kappa_{i}=\left|\left\{j \mid n+r_{j-1}-r_{j} \leqslant i-1, j=1,2, \ldots, m\right\}\right| \quad(i=1,2, \ldots, n),
$$

where

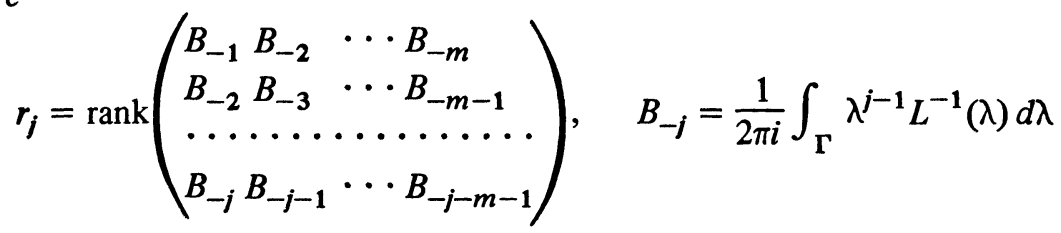

and $|\Omega|$ denotes the number of elements in the set $\Omega$.

The same formulas for $\kappa_{j}$ hold in case $\Gamma$ is the unit circle and the $B_{j}$ are the Fourier coefficients of $L^{-1}(\lambda)$ :

$$
L^{-1}(\lambda)=\sum_{j=-\infty}^{\infty} \lambda^{j} B_{j} \quad(|\lambda|=1)
$$

THEOREM 2. Suppose that all the eigenvalues of $L(\lambda)$ are inside $F^{+}$and $L(0)=I$. Then

where

$$
\kappa_{j}=\left|\left\{i \mid n+q_{i-1}-q_{i} \leqslant j-1, i=1,2, \ldots, n\right\}\right|
$$

$$
q_{i}=\operatorname{rank}\left(\begin{array}{l}
P C^{\nu} \\
P C^{\nu+1} \\
\vdots \\
P C^{\nu+i-1}
\end{array}\right), \quad C=\left(\begin{array}{cccccc}
0 & I & \cdot & \cdot & \cdot & 0 \\
\cdot & \cdot & \cdot & \cdot & \cdot & \cdot \\
0 & 0 & \cdot & \cdot & \cdot & I \\
-A_{m} & -A_{m-1} & \cdots & -A_{1}
\end{array}\right) \text {, }
$$

$\nu \geqslant 0$ is the minimal integer for which rank $C^{\nu}=\operatorname{rank} C^{\nu+1}$ and $P$ is the projector on the first $n$ coordinates.

If not all the eigenvalues of $L(\lambda)$ are inside $F^{+}$, then a similar result holds upon replacing $C$ by $C \widetilde{P}$, where $\widetilde{P}=(2 \pi i)^{-1} \int_{\Gamma}(\lambda I-C)^{-1} d \lambda$.

We now briefly sketch the main steps in the proofs.

At first without loss of generality in Theorem 1, we assume also that all the eigenvalues of $L(\lambda)$ are in $F^{+}$. This reduction is justified by some results from 
[4]. Now assuming that all the eigenvalues of $L(\lambda)$ are in $F^{+}$, let $J_{F}$ denote an $r \times r$ matrix in Jordan form, which has the same eigenvalues and the same elementary divisors as $L(\lambda)$. Let $X_{F}$ denote an $n \times r$ matrix with the eigenvectors and generalized eigenvectors of $L(\lambda)$ as columns arranged in correspondence with $J_{F}$. These eigenvectors can be chosen in a canonical way such that [4]

$$
\operatorname{rank} K_{m}=n m \text {, where } K_{j}=\left(\begin{array}{cc}
X_{F} \\
X_{F} & J_{F} \\
\vdots & \\
X_{F} & J_{F}^{j-1}
\end{array}\right) j=1,2, \ldots, m \text {. }
$$

The next step contains the proof of the equalities $q_{j}=r_{j}=\operatorname{rank} K_{j}$ for $j=$ $1,2, \ldots, m$.

The main step is the calculation of the indices $\kappa_{j}$ via the numbers rank $K_{j}$. It is based on an investigation of the subspaces $\operatorname{Ker} K_{j}^{*}$. Using the structure of these subspaces, we are able to construct a matrix polynomial $M(\lambda)$ with the same eigenvalues, eigenvectors and generalized eigenvectors as $L(\lambda)$ and with an additional important property: for some integers $\nu_{1} \leqslant v_{2} \leqslant \cdots \leqslant v_{n}$ the product $\left(\lambda^{v_{j}} \delta_{j k}\right) M(\lambda)$ is a matrix polynomial with an invertible leading coefficient. The connection between the numbers $\nu_{j}$ and $\operatorname{rank} K_{j}$ allows us to complete the proof.

The full proofs together with generalization for matrix valued functions and other results on indices will appear elsewhere.

\section{REFERENCES}

1. I. G. Gohberg and I. A. Feldman, Convolution equations and projection methods for their solution, Transl. Math. Monographs, Vol. 41, Amer. Math. Soc., Providence, R. I., 1974.

2. I. Gohberg, P. Lancaster and L. Rodman, Spectral analysis of matrix polynomials. I Canonical forms and divisors. II The resolvent form and spectral divisors, Linear Algebra and Appl. (to appear).

3. I. Gohberg and L. Rodman, On the spectral structure of monic matrix polynomials and the extension problem, Tel-Aviv Univ., Tel-Aviv, 1977 (preprint).

4. - On spectral analysis of nonmonic matrix and operator polynomials. I Reduction to monic polynomials. II Dependence of the finite spectral data, Tel-Aviv Univ., Tel-Aviv, 1977, (preprint).

5. N. I. Mushelisvili, Singular integral equations, Nordhoff, Groningen, 1953.

DEPARTMENT OF MATHEMATICAL SCIENCES, TEL-AVIV UNIVERSITY, RAMATAVIV, TEL-AVIV, ISRAEL (Current address of I. Gohberg and L. Rodman)

DEPARTMENT OF MATHEMATICS, TECHNION, HAIFA, ISRAEL (Current address of L. Lerer) 